\title{
NON-ESSENTIAL CLUSTER VALUES AND NORMAL FUNCTIONS
}

\author{
C. L. BELNA*
}

\section{Introduction}

We consider continuous functions $f$ which map the open unit disk $D$ into the Riemann sphere $W$. For a point $\zeta$ on the unit circle $C$, we say that $\chi$ is a chord at $\zeta$ if $\chi$ is a chord of $C$ having one endpoint at $\zeta$ and that $\Delta$ is a Stolz angle at $\zeta$ if $\Delta$ is a Stolz angle with vertex $\zeta$. Suppose $S$ denotes either a chord at $\zeta$, a Stolz angle at $\zeta$, or $D$. Then, letting $\sigma$ denote the chordal metric on $W$ and setting

$$
S_{r}=S \cap\{z \in D:|z-\zeta|<r\} \quad(r>0),
$$

we define the cluster set $C(f, \zeta, S)$ of $f$ at $\zeta$ relative to $S$ and the essential cluster set $C_{e}(f, \zeta, S)$ of $f$ at $\zeta$ relative to $S$ as follows: the point $w^{*} \in W$ is in $C(f, \zeta, S)$ if, for every $\varepsilon>0$ and every $r>0$,

$$
S_{r} \cap f^{-1}\left(\left\{w \in W: \sigma\left(w, w^{*}\right)<\varepsilon\right\}\right) \neq \phi ;
$$

whereas $w^{*}$ is in $C_{e}(f, \zeta, S)$ if, for every $\varepsilon>0$,

$$
\limsup _{r \rightarrow 0} \frac{m\left[S_{r} \cap f^{-1}\left(\left\{w \in W: \sigma\left(w, w^{*}\right)<\varepsilon\right\}\right)\right]}{m S_{r}}>0,
$$

where $m$ denotes linear Lebesgue measure $m_{1}$ if $S$ is a chord at $\zeta$ and denotes 2-dimensional Lebesgue measure $m_{2}$ if $S$ is either a Stolz angle at $\zeta$ or $D$. The abbreviated notations $C(f, \zeta)$ and $C_{e}(f, \zeta)$ are used in place of $C(f, \zeta, D)$ and $C_{e}(f, \zeta, D)$. We remark that both $C(f, \zeta, S)$ and $C_{e}(f, \zeta, S)$ are closed subsets of $W$ with $C_{e}(f, \zeta, S) \subset C(f, \zeta, S)$ and that

$$
\lim _{r \rightarrow 0} \frac{m\left[S_{r} \cap f^{-1}(G)\right]}{m S_{r}}=1
$$

Received January 8, 1971.

* Research performed in part at the U.S.A.F. Aerospace Research Laboratories while in the capacity of an Ohio State University Research Foundation Research Analyst under Contract F33615-67-C-1758. 
for each open subset $G$ of $W$ containing $C_{e}(f, \zeta, S)$. (Note that the latter fact is trivially true for open sets $G$ containing $C(f, \zeta, S)$.)

The object of our study is the open set $C(f, \zeta, S)-C_{e}(f, \zeta, S)$, which we call the set of non-essential cluster values of $f$ at $\zeta$ relative to $S$. In section 2 (3) we give a necessary condition for a point $w$ to be a non-essential cluster value of $f$ at $\zeta$ relative to a chord (Stolz angle) at $\zeta$. In both of these sections we make use of the following lemma of Lappan [3, Lemma 2, p. 46] (see also Rung [7, p. 424]) concerning the non-Euclidean hyperbolic distance between points $a$ and $b$ in $D$ given by

$$
\rho(a, b)=\tanh ^{-1}\left(\left|\frac{a-b}{1-\bar{a} b}\right|\right) .
$$

LEMMA L. If $a$ and $b$ are in $D$ with $\rho(a, b)=M$, then

$$
\tanh M \leq \frac{|a-b|}{1-|a|} \leq \frac{2 \tanh M}{1-\tanh M} .
$$

We show in section 4 that normal functions have neither non-essential chordal cluster values nor non-essential angular cluster values. On the other hand, in the last section we exhibit a normal function having non-essential cluster values at almost every $\zeta \in C$ relative to $D$.

\section{Chordal Cluster Sets}

For each $a \in D$ and each $M>0$, we set

$$
D(a, M)=\{z \in D: \rho(a, z)<M\}
$$

and we denote the boundary of $D(a, M)$ by $\partial D(a, M)$.

LEMMA 1. Suppose $\left\{a_{n}\right\}$ is a sequence of points lying on the chord $\chi$ at $\zeta \in C$ with $a_{n} \rightarrow \zeta$. Then, for each $M>0$,

$$
\limsup _{r \rightarrow 0} \frac{m_{1}\left[\chi_{r} \cap \bigcup_{n=1}^{\infty} D\left(a_{n}, M\right)\right]}{m_{1} \chi_{r}}>0 .
$$

Proof. Choose a number $M>0$. Denote by $b_{n}$ the point of $\chi \cap$ $\partial D\left(a_{n}, M\right)$ that is furthest from $\zeta$ (in the Euclidean sense). Setting $r_{n}=\left|b_{n}-\zeta\right|$, we have

$$
\frac{m_{1}\left[\chi_{r_{n}} \cap D\left(a_{n}, M\right)\right]}{m_{1} \chi_{r_{n}}}>\frac{\left|a_{n}-b_{n}\right|}{\left|b_{n}-\zeta\right|} .
$$

Since $\left\{b_{n}\right\} \subset \chi$, there exists a constant $K>0$ with $\left(1-\left|b_{n}\right|\right) /\left|b_{n}-\zeta\right|>K$ 
for all $n$. Also, by Lemma $\mathrm{L},\left|a_{n}-b_{n}\right| /\left(1-\left|b_{n}\right|\right) \geq \tanh M$ for each $n$. Consequently

$$
\liminf _{n \rightarrow \infty} \frac{m_{1}\left[\chi_{r_{n}} \cap D\left(a_{n}, M\right)\right]}{m_{1} \chi_{r_{n}}} \geq K \tanh M
$$

which clearly implies the conclusion of the lemma.

A sequence $\left\{a_{n}\right\} \subset D$ is said to be close to the sequence $\left\{b_{n}\right\} \subset D$ if $\rho\left(a_{n}, b_{n}\right) \rightarrow 0$ as $n \rightarrow \infty$.

THEOREM 1. Let $f: D \rightarrow W$ be continuous, let $\chi$ be a chord at $\zeta \in C$, and suppose $w_{0} \in C(f, \zeta, \chi)-C_{e}(f, \zeta, \chi)$. If $\left\{a_{n}\right\}$ is a sequence of points on $\chi$ with $a_{n} \rightarrow \zeta$ and $f\left(a_{n}\right) \rightarrow w_{0}$, then there exists a sequence $\left\{b_{n}\right\} \subset \chi$ which is close to a subsequence of $\left\{a_{n}\right\}$ and for which $\left\{f\left(b_{n}\right)\right\}$ converges to a point of $C_{e}(f, \zeta, \chi)$.

Proof. Set $G_{j}=\left\{w \in W: \sigma\left(w, C_{e}(f, \zeta, \chi)\right)<1 / j\right\}$ and choose positive integers $N$ and $J$ such that $f\left(a_{n}\right) \notin \bar{G}_{j}$ (the closure of $G_{j}$ ) for $n>N$ and $j>J$. Set

$$
M(n, j)=\max \left\{M: f\left(\chi \cap D\left(a_{n}, M\right)\right) \cap G_{j}=\phi\right\},
$$

and suppose there exists a $j_{0}>J$ for which

$$
\limsup _{n \rightarrow \infty} M\left(n, j_{0}\right)=M_{j_{0}}>0 \text {. }
$$

This implies the existence of a subsequence $\left\{a_{n}^{\prime}\right\}$ of $\left\{a_{n}\right\}$ such that $f(\chi \cap$ $\left.D\left(a_{n}^{\prime}, M_{j_{0}} / 2\right)\right) \cap G_{j_{0}}=\phi$ for each $n$. Then, by Lemma 1 ,

$$
\limsup _{r \rightarrow 0} \frac{m_{1}\left[\chi_{r} \cap \bigcup_{n=1}^{\infty} D\left(a_{n}^{\prime}, M_{j_{0}} / 2\right)\right]}{m_{1} \chi_{r}}>0 ;
$$

and hence

$$
\lim _{r \rightarrow 0} \frac{m_{1}\left[\chi_{r} \cap f^{-1}\left(G_{j_{0}}\right)\right]}{m_{1} \chi_{r}} \neq 1
$$

in violation of $C_{e}(f, \zeta, \chi) \subset G_{j_{0}}$. It follows that, for each $j>J$, $\lim M(n, j)=0$. Thus for each $j>J$ there exist an integer $n_{j}$ and a point $b_{j} \in \chi$ for which $\left|a_{n_{j}}-\zeta\right|<1 / j, \rho\left(a_{n_{j}}, b_{j}\right)<1 / j$ and $f\left(b_{j}\right) \in G_{j}$. Then since each convergent subsequence of $\left\{f\left(b_{j}\right)\right\}$ converges to a point of $C_{e}(f, \zeta, \chi)$, the theorem is proved. 
A chord $\chi$ at $\zeta \in C$ is called a segment of Julia for a function $f$ at $\zeta$ provided $f$ assumes all values of $W$ except possibly two in each Stolz angle at $\zeta$ meeting $\chi$.

COROLlary 1. Let $f$ be a meromorphic function in $D$, and let $\chi$ be a chord at $\zeta \in C$. If $C_{e}(f, \zeta, \chi) \neq C(f, \zeta, \chi)$, then $\chi$ is a segment of Julia for $f$ at $\zeta$.

Proof. Suppose $C_{e}(f, \zeta, \chi) \neq C(f, \zeta, \chi)$. Applying Theorem 1 we obtain sequences $\left\{a_{n}\right\},\left\{b_{n}\right\} \subset \chi$ and distinct complex values $\alpha$ and $\beta$ for which $\rho\left(a_{n}, b_{n}\right) \rightarrow 0, f\left(a_{n}\right) \rightarrow \alpha$ and $f\left(b_{n}\right) \rightarrow \beta$. According to Lappan [3, Theorem 4, p. 44], for each value $\delta \in W$ with perhaps two exceptions, there exists a sequence $\left\{z_{k}^{\delta}\right\}$ close to a subsequence of $\left\{a_{n}\right\}$ with $f\left(z_{k}^{\delta}\right)=\delta$ for each $k$. Then, for any Stolz angle $\Delta$ at $\zeta$ meeting $\chi$, each of the sequences $\left\{z_{k}^{\delta}\right\}$ has a terminal subsequence that lies in $\Delta$ and the corollary is proved.

The outer angular cluster set of $f$ at $\zeta \in C$ is the set

$$
C_{\mathscr{A}}(f, \zeta)=\bigcup_{\Delta} C(f, \zeta, \Delta)
$$

where $\Delta$ ranges over all Stolz angles at $\zeta$. Since $C(f, \zeta, \Delta)=W$ for each Stolz angle $\Delta$ at $\zeta$ meeting a segment of Julia for $f$ at $\zeta$, we have the following result.

CoRollary 2. Let $f$ be a meromorphic function in $D$, and let $\zeta$ be a point of $C$. If $C_{s}(f, \zeta) \neq W$, then $C_{e}(f, \zeta, \chi)=C(f, \zeta, \chi)$ for every chord $\chi$ at $\zeta$.

\section{Angular Cluster Sets}

We start with a simple lemma.

LEMMA 2. Let a be a point of $D$, let $\chi$ be the chord at $\zeta \in C$ that passes through a, and let $M$ be a positive number. If $Q$ represents either component of $D(\alpha, M)-\chi$, then

$$
m_{2} Q \geq K_{M}(1-|a|)^{2}
$$

where $K_{M}=[\arcsin (\operatorname{sech} M)-\tanh M \operatorname{sech} M] \tanh ^{2} M>0$.

Proof. For $a=0, m_{2} Q=(\pi / 2) \tanh ^{2} M>K_{M}$. Suppose $a \neq 0$. Let $A$ denote the line segment joining $a$ to the origin, and let $\chi^{*}$ be the chord of $C$ that passes through $a$ and is orthogonal to $A$. Then let $T$ 
be the component of $D(a, M)-\chi^{*}$ that is separated from the origin by $\chi^{*}$. Clearly $m_{2} Q \geq m_{2} T$. Through elementary calculations we find that

$$
m_{2} T=\tau[|a|, M](1-|a|)^{2}
$$

with

$$
\tau[|a|, M]=\left(\frac{1+|a|}{|a|}\right)^{2}\left(\frac{\lambda}{1-\lambda^{2}}\right)^{2}\left[\arcsin \left(\sqrt{1-\lambda^{2}}\right)-\lambda \sqrt{1-\lambda^{2}}\right]
$$

where $\lambda=|a| \tanh M$. Finally, it is easy to see that $\tau[|a|, M] \geq K_{M}$ and the proof is complete.

LEMma 3. Let $\left\{a_{n}\right\}$ be a sequence of points in the Stolz angle $\Delta$ at $\zeta \in C$ with $a_{n} \rightarrow \zeta$. Then, for each $M>0$,

$$
\limsup _{r \rightarrow 0} \frac{m_{2}\left[\Delta_{r} \cap \bigcup_{n=1}^{\infty} D\left(a_{n}, M\right)\right]}{m_{2} \Delta_{r}}>0 .
$$

Proof. Choose a number $M>0$. Since $\left\{a_{n}\right\} \subset \Delta$, there exists a number $M^{*}\left(0<M^{*} \leq M\right)$ such that the set $D\left(a_{n}, M^{*}\right) \cap(D-\Delta)$ is connected for each $n$. Let $\chi_{n}$ denote the chord at $\zeta$ that passes through the point $a_{n}$, and let $D_{n}^{1}$ and $D_{n}^{2}$ denote the components of $D\left(a_{n}, M^{*}\right)-\chi_{n}$ with $m_{2} D_{n}^{1}$ $\leq m_{2} D_{n}^{2}$. It is clear that, for each $n$, either $D_{n}^{1} \subset \Delta$ or $D_{n}^{2} \subset \Delta$. Denote by $b_{n}$ the point of $\chi_{n} \cap \partial D\left(a_{n}, M^{*}\right)$ that is furthest from $\zeta$ (in the Euclidean sense), and set $r_{n}=\left|b_{n}-\zeta\right|$. If $E_{n}$ denotes the region obtained by reflecting $D_{n}^{1}$ across $\chi_{n}$, it is evident that

$$
E_{n} \subset D_{n}^{2} \cap\left\{z \in D:|z-\zeta|<r_{n}\right\}
$$

and that $m_{2} E_{n}=m_{2} D_{n}^{1}$. Thus

$$
m_{2}\left[\Delta_{r_{n}} \cap D\left(a_{n}, M^{*}\right)\right] \geq m_{2} D_{n}^{1} .
$$

Applying Lemma 2 we obtain

$$
m_{2}\left[\Delta_{r_{n}} \cap D\left(a_{n}, M^{*}\right)\right] \geq K_{M^{*}}\left(1-\left|a_{n}\right|\right)^{2} .
$$

Then, using $\alpha$ to denote the angular opening of $\Delta$, we have

$$
\frac{m_{2}\left[\Delta_{r_{n}} \cap D\left(a_{n}, M^{*}\right)\right]}{m_{2} \Delta_{r_{n}}} \geq\left(2 \alpha^{-1} K_{M^{*}}\right)\left(\frac{1-\left|a_{n}\right|}{\left|b_{n}-\zeta\right|}\right)^{2} .
$$

It follows from Lemma $\mathrm{L}$ that there exists a constant $A>0$ such that 
$\left(1-\left|a_{n}\right|\right) /\left(1-\left|b_{n}\right|\right) \geq A$ for all $n$; and, since $\left\{b_{n}\right\} \subset \Delta$, there exists a constant $B>0$ with $\left(1-\left|b_{n}\right|\right) /\left|b_{n}-\zeta\right| \geq B$ for all $n$. Hence

$$
\liminf _{n \rightarrow \infty} \frac{m_{2}\left[\Delta_{r_{n}} \cap D\left(a_{n}, M^{*}\right)\right]}{m_{2} \Delta_{r_{n}}} \geq(A B)^{2} \alpha^{-1} K_{M^{*}}>0,
$$

and the conclusion of the lemma now follows.

We now give the analogue of Theorem 1 for Stolz angles.

THEOREM 2. Let $f: D \rightarrow W$ be continuous, let $\Delta$ be a Stolz angle at $\zeta \in C$, and suppose $w_{0} \in C(f, \zeta, \Delta)-C_{e}(f, \zeta, \Delta)$. If $\left\{a_{n}\right\}$ is a sequence of points in $\Delta$ with $a_{n} \rightarrow \zeta$ and $f\left(a_{n}\right) \rightarrow w_{0}$, then there exists a sequence $\left\{b_{n}\right\} \subset \Delta$ which is close to a subsequence of $\left\{a_{n}\right\}$ and for which $\left\{f\left(b_{n}\right)\right\}$ converges to a point of $C_{e}(f, \zeta, \Delta)$.

The proof of Theorem 2 is obtained by using Lemma 3 in place of Lemma 1 and replacing $\chi$ by $\Delta$ in the proof of Theorem 1 . Also the proofs of the following corollaries are similar to those of the corollaries of Theorem 1 .

COROLlaRY 1. Let $f$ be a meromorphic function in $D$, and let $\Delta$ be a Stolz angle at $\zeta \in C$. If $C_{e}(f, \zeta, \Delta) \neq C(f, \zeta, \Delta)$, then $f$ assumes all values on $W$ except possibly two in each Stolz angle $\Delta^{*}$ at $\zeta$ containing $\bar{\Delta}$.

COROLlaRY 2. Let $f$ be a meromorphic function in $D$, and let $\zeta$ be a point of $C$. If $C_{s}(f, \zeta) \neq W$, then $C_{e}(f, \zeta, \Delta)=C(f, \zeta, \Delta)$ for each Stolz angle $\Delta$ at $\zeta$.

\section{Applications to Normal Functions}

Let $\mathscr{T}$ denote the collection of all one-one conformal mappings of $D$ onto $D$. A continuous function $f: D \rightarrow W$ is said to be normal if the family of functions $\{f(T(z))\}_{T(z) \in \mathcal{F}}$ is normal in $D$ in the sense of Montel.

THEOREM 3. Suppose the continuous function $f: D \rightarrow W$ is normal. Then, for each $\zeta \in C$, (1) $C_{e}(f, \zeta, \chi)=C(f, \zeta, \chi)$ for each chord $\chi$ at $\zeta$ and (2) $C_{e}(f, \zeta, \Delta)=C(f, \zeta, \Delta)$ for each Stolz angle $\Delta$ at $\zeta$.

Proof. Assume $C_{e}(f, \zeta, \chi) \neq C(f, \zeta, \chi)$ for some $\zeta \in C$ and some chord $\chi$ at $\zeta$. By Theorem 1 there exist sequences $\left\{a_{n}\right\},\left\{b_{n}\right\} \subset \chi$ and distinct complex values $\alpha$ and $\beta$ for which $\rho\left(a_{n}, b_{n}\right) \rightarrow 0, f\left(a_{n}\right) \rightarrow \alpha$ and $f\left(b_{n}\right) \rightarrow \beta$. According to Lappan [4, Theorem 2, p. 156], $f$ is non-normal in violation of the hypothesis; and (1) is proved. The proof of (2) is similar. 
The converse of Theorem 3 is not true, as the following theorem shows.

THEOREM 4. There exists a non-normal continuous function $f: D \rightarrow$ $W$ such that, for each $\zeta \in C$, (1) $C_{e}(f, \zeta, \chi)=C(f, \zeta, \chi)$ for each chord $\chi$ at $\zeta$ and (2) $C_{e}(f, \zeta, \Delta)=C(f, \zeta, \Delta)$ for each Stolz angle $\Delta$ at $\zeta$.

Proof. Define the sets

$$
A=\{z \in D:|z-3 / 4| \leq 1 / 4\}
$$

and

$$
B=\{z \in D:|z-1 / 2| \geq 1 / 2\} .
$$

Let $\left\{a_{n}\right\}$ and $\left\{b_{n}\right\}$ be disjoint sequences of points in $D-(A \cup B)$ with $a_{n} \rightarrow 1$ and $\rho\left(a_{n}, b_{n}\right) \rightarrow 0$. Define the continuous function $F$ on $A \cup B \cup$ $\left\{a_{n}\right\} \cup\left\{b_{n}\right\}$ by

$$
F(z)= \begin{cases}w_{1} & \text { for } z \in A \cup B \cup\left\{a_{n}\right\} \\ w_{2} & \text { for } z \in\left\{b_{n}\right\}\end{cases}
$$

where $w_{1}$ and $w_{2}$ are distinct points of $W$. The function $F$ can be extended to a continuous function $f: D \rightarrow W$. It follows from the result of Lappan cited in the proof of Theorem 3 that $f$ is non-normal. Furthermore, for each $\zeta \in C$,

$$
C(f, \zeta, S)=\left\{w_{1}\right\}=C_{e}(f, \zeta, S)
$$

where $S$ denotes an arbitrary Stolz angle or chord at $\zeta$. Hence the theorem is proved.

We now show that Theorem 3 is not true if the condition that $f$ is normal is removed, even if $f$ is assumed to be holomorphic.

THEOREM 5. The function

$$
F(z)=\prod_{j=1}^{\infty}\left\{1-\left(\frac{z}{1-n_{j}^{-1}}\right)^{n_{j}^{2}}\right\} \quad\left(n_{j}=3^{j}\right)
$$

is holomorphic in $D$ and has the properties: (1) for nearly every $\zeta \in C$, $C_{e}\left(F, \zeta, \rho_{\zeta}\right) \neq C\left(F, \zeta, \rho_{\zeta}\right)$ where $\rho_{\zeta}$ denotes the chord at $\zeta$ which forms $a$ diameter of $C$, and (2) for almost every $\zeta \in C, C_{e}(F, \zeta, \Delta) \neq C(F, \zeta, \Delta)$ where $\Delta$ denotes an arbitrary Stolz angle at $\zeta$.

Proof. Bagemihl and Seidel [1] have shown that $F(z)$ is holomorphic 
in $D$ and that $F(z) \rightarrow \infty$ as $|z| \rightarrow 1$ through a region $\Omega$ which is described as follows: for $j=1,2,3, \cdots$ and $\nu=0,1, \cdots, n_{j}^{2}-1$ set

$$
z_{j_{\nu}}=\left(1-n_{j}^{-1}\right) e^{2 \pi i \nu / n_{j}^{2}}
$$

and

$$
\Gamma_{j_{\nu}}=\left\{z:\left|z-z_{j_{\nu}}\right| \leq r_{j}\right\}
$$

where $r_{j}=1 / n_{j}^{4}$. Then $\Omega$ is the region obtained by deleting all the $\operatorname{disks} \Gamma_{j_{\nu}}$ from $D$.

Since each point of $C$ is a limit point of the set $\left\{z_{j_{v}}\right\}$ of zeros of $F(z), 0 \in C(F, \zeta)$ for every $\zeta \in C$; and it follows from a theorem of Collingwood [6, p. 66] that $0 \in C\left(F, \zeta, \rho_{\zeta}\right)$ for nearly every $\zeta \in C$. Also, as a consequence of the uniqueness theorem of Lusin and Privaloff [6, p. 72] and Plessner's theorem [6, p. 70], for almost every $\zeta \in C, C(F, \zeta, \Delta)$ $=W$ for each Stolz angle $\Delta$ at $\zeta$. That $F(z)$ has properties (1) and (2) now follows from the next two lemmas.

LeMma 4. $C_{e}(F, \zeta, \chi)=\{\infty\}$ for every $\zeta \in C$ and every chord $\chi$ at $\zeta$.

Proof. Let $\zeta$ be a point of $C$ and let $\chi$ be a chord at $\zeta$. For $1 / n_{k+1} \leq r \leq 1 / n_{k}$ we have

$$
\begin{aligned}
\frac{m_{1}\left[\chi_{r} \cap(D-\Omega)\right]}{m_{1} \chi_{r}} & \leq n_{k+1} \sum_{j=k}^{\infty} \frac{2}{n_{j}^{4}} \\
& \leq 6 \sum_{j=k}^{\infty} \frac{1}{n_{j}^{3}} \rightarrow 0 \text { as } k \rightarrow \infty .
\end{aligned}
$$

Hence

$$
\lim _{r \rightarrow 0} \frac{m_{1}\left[\chi_{r} \cap(D-\Omega)\right]}{m_{1} \chi_{r}}=0,
$$

which implies $C_{e}(F, \zeta, \chi)=\{\infty\}$.

Lemma 5. $C_{e}(F, \zeta, \Delta)=\{\infty\}$ for every $\zeta \in C$ and every Stolz angle $\Delta$ at $\zeta$.

Proof. Choose a point $\zeta \in C$ and let $\Delta$ be a Stolz angle at $\zeta$ with angular opening $\alpha$. For $1 / n_{k+1} \leq r \leq 1 / n_{k}$ we have

$$
\begin{aligned}
\frac{m_{2}\left[\Delta_{r} \cap(D-\Omega)\right]}{m_{2} \Delta_{r}} & \leq 2 \alpha^{-1} n_{k+1}^{2} \sum_{j=k}^{\infty} n_{j}^{2}\left[\pi\left(1 / n_{j}^{4}\right)^{2}\right] \\
& \leq 18 \alpha^{-1} \pi \sum_{j=k}^{\infty} \frac{1}{n_{j}^{4}} \rightarrow 0 \text { as } \quad k \rightarrow \infty .
\end{aligned}
$$


Consequently

$$
\lim _{r \rightarrow 0} \frac{m_{2}\left[\Delta_{r} \cap(D-\Omega)\right]}{m_{2} \Delta_{r}}=0,
$$

and so $C_{e}(F, \zeta, \Delta)=\{\infty\}$.

\section{Total Cluster Sets}

If the analogue of Theorem 1 (or Theorem 2) for $C(f, \zeta)$ and $C_{e}(f, \zeta)$ were true, it would easily follow that these two sets are always equal for normal functions. Thus the next theorem shows that no such analogue exists. (In this section $m$ denotes linear measure.)

THEOREM 6. There exists a bounded holomorphic function $g$ in $D$ for which

$$
m\left\{\zeta \in C: C_{e}(g, \zeta)=C(g, \zeta)\right\}=0 .
$$

To establish the existence of such a function, we make use of the following portion of a result of Goffman and Sledd [2, Theorem 2]. (We note that their proof is for real-valued functions in the upper half plane, but only slight modifications are needed to obtain a proof for complexvalued functions in $D$.)

THEOREM GS. For each $\zeta \in C$ let $\rho_{\zeta}$ denote the chord at $\zeta$ which forms a diameter of $C$. If $f: D \rightarrow W$ is measurable, then

$$
m\left\{\zeta \in C: C_{e}(f, \zeta) \subset C_{e}\left(f, \zeta, \rho_{\zeta}\right)\right\}=2 \pi .
$$

Proof of Theorem 6. It follows from a theorem of Littlewood [5, p. 172] that there exists a bounded holomorphic function $g$ in $D$ for which there exists a subset $E$ of $C$ with $m E=2 \pi$ and $C\left(g, \zeta, \rho_{\zeta}\right) \neq C(g, \zeta)$ for each $\zeta \in E$. By Theorem GS there exists a subset $F$ of $C$ with $m F$ $=2 \pi$ and $C_{e}(g, \zeta) \subset C_{e}\left(g, \zeta, \rho_{\zeta}\right)$ for each $\zeta \in F$. Then in view of Theorem 3 we have

$$
C_{e}(g, \zeta) \subset C_{e}\left(g, \zeta, \rho_{\zeta}\right)=C\left(g, \zeta, \rho_{\zeta}\right) \subseteq C(g, \zeta)
$$

for each $\zeta \in E \cap F$, and the theorem is proved. 


\section{REFERENCES}

[1] F. Bagemihl and W. Seidel, Some boundary properties of analytic functions, Math. Z., 61 (1954), 186-199.

[ 2 ] Casper Goffman and W. T. Sledd, Essential Cluster Sets, J. London Math. Soc., (2), 1 (1969), 295-302.

[3] Peter Lappan, Some sequential properties of normal and non-normal functions with applications to automorphic functions, Comm. Math. Univ. Sancti Pauli, 12 (1964), 41-57.

[ 4 ] - Some results on harmonic normal functions, Math. Z., 90 (1965), 155-159.

[5] J. E. Littlewood, Mathematical Notes (4): On a theorem of Fatou, London Math. J., 2 (1947), 172-176.

[ 6 ] K. Noshiro, Cluster sets, Berlin, 1960.

[ 7 ] D. C. Rung, Behavior of holomorphic functions in the unit disk on arcs of positive hperbolyic diameter, J. Math. Kyoto Univ., 8-3 (1968), 417-464.

Wright State University

Dayton, Ohio 45431 